\title{
Effective Descent Morphisms in Categories of Lax Algebras
}

\author{
MARIA MANUEL CLEMENTINO ${ }^{1}$ and DIRK HOFMANN ${ }^{2}$ \\ ${ }^{1}$ Departamento de Matemática, Universidade de Coimbra, 3001-454 Coimbra, Portugal. \\ e-mail:mmc@mat.uc.pt \\ ${ }^{2}$ Departamento de Matemática, Universidade de Aveiro, 3810-193 Aveiro, Portugal. \\ e-mail:dirk@mat.ua.pt
}

\begin{abstract}
In this paper we investigate effective descent morphisms in categories of reflexive and transitive lax algebras. We show in particular that open and proper maps are of effective descent, result that extends the corresponding results for the category of topological spaces and continuous maps.
\end{abstract}

Mathematics Subject Classifications (2000): 18D05, 18C20, 18D10.

Key words: monad, effective descent morphism, lax algebra.

\section{Introduction}

A morphism $p: E \rightarrow B$ in a category $\mathbf{C}$ with pullbacks is called an effective descent morphism if it allows a description of structures over the base $B$ as algebras on structures over the extension $E$ of $B$. Here the meaning of "structure over $B$ " might depend on the category $\mathbf{C}$; however, in this paper we define it simply to be a morphism with codomain $B$. In that particular case $p: E \rightarrow B$ is of effective descent if and only if the pullback functor $p^{*}:(\mathbf{C} \downarrow B) \rightarrow(\mathbf{C} \downarrow E)$ is monadic. In locally cartesian closed categories effective descent morphisms are easy to describe: they are exactly the regular epimorphisms. Such a characterization is far from being valid in an arbitrary category; in general it can be quite a hard problem to find necessary and sufficient conditions for a morphism to be of effective descent (see, for instance, [13] for the topological case). In order to obtain such conditions, it is often useful to embed our category into a category which has an easy description of effective descent morphisms, and then apply the pullback criterion of Theorem 2.1 below; this will be the basic technique of this paper.

Following a suggestion of George Janelidze, we investigate effective descent morphisms in categories of reflexive and transitive lax algebras $\operatorname{Alg}(T ; \mathbf{V})$ when $\mathbf{V}$ is a lattice, providing this way a unified treatment of descent theory for various categories. In particular, we characterize effective descent morphisms between premetric and metric spaces and, moreover, show that (suitably defined) open and proper 
maps are effective descent morphisms in $\operatorname{Alg}(\mathrm{T} ; \mathbf{V})$, encompassing the results for topological spaces obtained by Moerdijk [11, 12] and Sobral (see [14]).

\section{Preliminaries}

Throughout we will be working in the setting described in [6], restricted to the case of a non-degenerated lattice $\mathbf{V}$. More precisely,

- $\quad \mathbf{V}$ is a complete symmetric monoidal closed (non-degenerated, i.e. with $0 \neq 1$ ) lattice, with tensor product $\otimes$ and unit $I$, and

- $\mathbf{T}=(T, e, m)$ is a monad on Set lax-extended to $\operatorname{Mat}(\mathbf{V})$.

We recall that $\operatorname{Mat}(\mathbf{V})$ is the bicategory with sets as objects, with 1-cells $r$ : $X \nrightarrow Y$ given by $X \times Y$-matrices in $\mathbf{V}$ (that is, $r$ is a map $X \times Y \rightarrow \mathbf{V}$ ), and with 2-cells determined by the componentwise order in $\mathbf{V}$ :

$$
(r: X \nrightarrow Y) \leq(s: X \nrightarrow Y) \text { if } \forall(x, y) \in X \times Y r(x, y) \leq s(x, y) \text { in } \mathbf{V} .
$$

Composition of 1-cells is given by matrix multiplication, so that, for $r: X \nrightarrow Y$ and $s: Y \nrightarrow Z$,

$$
(s \cdot r)(x, z)=\bigvee_{y \in Y} r(x, y) \otimes s(y, z) .
$$

$\operatorname{Mat}(\mathbf{V})$ has a natural involution, given by matrix transposition:

$$
\text { for } r: X \nrightarrow Y, r^{\circ}: Y \nrightarrow X \text { is defined by } r^{\circ}(y, x):=r(x, y) \text {. }
$$

The category Set can be naturally embedded into $\operatorname{Mat}(\mathbf{V})$, assigning to each map $f: X \rightarrow Y$ the matrix with $(x, y)$-entry $I$ in case $y=f(x)$ and 0 otherwise. By an extension of the Set-monad $\mathrm{T}=(T, e, m)$ into $\operatorname{Mat}(\mathbf{V})$ we mean a lax functor $T: \operatorname{Mat}(\mathbf{V}) \rightarrow \operatorname{Mat}(\mathbf{V})$ that extends the endofunctor $T$ of Set and such that the natural transformations $e$ and $m$ become op-lax; this means that:

- $T s \cdot T r \leq T(s \cdot r)$,

- $e_{Y} \cdot r \leq T r \cdot e_{X}$ and

- $m_{Y} \cdot T^{2} r \leq T r \cdot m_{X}$

for all $r: X \nrightarrow Y$ and $s: Y \nrightarrow Z$ in $\operatorname{Mat}(\mathbf{V})$. In addition we require that $T$ preserves the involution. A possible construction of such an extension is discussed in [4]. As it is observed in [3], from this property it follows that $T$ is functorial with respect to composition with maps on the right.

We say that a square diagram

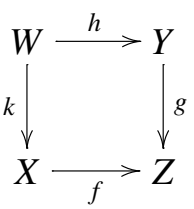


in Set satisfies the Beck-Chevalley condition (BC) if

$$
g^{\circ} \cdot f=h \cdot k^{\circ} .
$$

(We point out that a BC-square does not need to be commutative.) We will also say that a functor $T:$ Set $\rightarrow$ Set preserves Beck-Chevalley squares (or simply $B C$-squares) if, whenever diagram (1) satisfies (BC), its image by $T$ satisfies (BC) as well, i.e.

$$
T g^{\circ} \cdot T f=T h \cdot T k^{\circ} .
$$

We denote by $\operatorname{Alg}(T, e ; \mathbf{V})$ the category of reflexive lax algebras and lax homomorphisms; that is, its objects are pairs $(X, a)$ where $X$ is a set and $a: T X \nrightarrow X$ is a 1-cell in $\operatorname{Mat}(\mathbf{V})$ such that

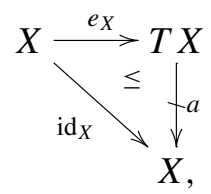

and its morphisms $(X, a) \rightarrow(Y, b)$ are maps $f: X \rightarrow Y$ such that

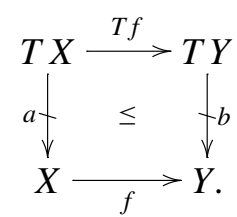

The category $\operatorname{Alg}(T ; \mathbf{V})$ is the full subcategory of $\operatorname{Alg}(T, e ; \mathbf{V})$ whose objects $(X, a)$ satisfy in addition

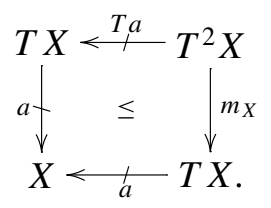

These categories are both topological over Set, hence in particular complete and cocomplete. Moreover, $\operatorname{Alg}(\mathrm{T} ; \mathbf{V})$ is a reflective subcategory of $\operatorname{Alg}(T, e ; \mathbf{V}$ ) (see [3] and [6] for details).

\section{Effective Descent Morphisms in $\operatorname{Alg}(T ; \mathbf{V})$}

In order to identify effective descent morphisms in a category, it is often useful to embed it in another category whose effective descent morphisms are better behaved. The following result, that can be found in [9], gives one such technique.

THEOREM 2.1. Let $\mathbf{A}$ and $\mathbf{B}$ be categories such that 
(a) $\mathbf{B}$ has pullbacks and coequalizers and $\mathbf{A}$ is a full subcategory of $\mathbf{B}$ closed under pullbacks, and

(b) every regular epimorphism in $\mathbf{B}$ is an effective descent morphism.

Then a morphism $p: E \rightarrow B$ in $\mathbf{A}$, which is of effective descent in $\mathbf{B}$, is an effective descent morphism in $\mathbf{A}$ if and only if

$$
E \times{ }_{B} A \in \mathbf{A} \Rightarrow A \in \mathbf{A}
$$

holds for every pullback

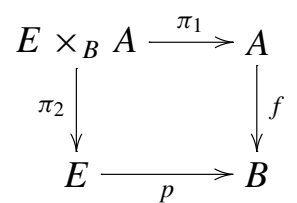

in $\mathbf{B}$.

This theorem can be applied to $\mathbf{A}=\operatorname{Alg}(\mathrm{T} ; \mathbf{V}) \hookrightarrow \operatorname{Alg}(T, e ; \mathbf{V})=\mathbf{B}$, whenever condition (b) is fulfilled, since (a) follows from the last remark of Section 1. Since regular epimorphisms are effective descent morphisms in a category whenever it is locally cartesian closed (see, for instance, [13]), the following result will be essential in the sequel.

THEOREM 2.2 ([5]). If the Set-functor $T$ preserves $B C$-squares and $\mathbf{V}$ is complete and locally cartesian closed, then also $\operatorname{Alg}(T, e ; \mathbf{V})$ is locally cartesian closed.

Since a complete lattice is locally cartesian closed if and only if it is cartesian closed, the assumption on $\mathbf{V}$ in the theorem can be replaced by the property of $\mathbf{V}$ being a Heyting algebra.

We will also use the characterization of regular epimorphisms in $\operatorname{Alg}(T, e ; \mathbf{V})$ below.

PROPOSITION 2.3 ([3]). A morphism $f:(X, a) \rightarrow(Y, b)$ in $\operatorname{Alg}(T, e ; \mathbf{V})$ is a regular epimorphism if and only if $b=f \cdot a \cdot(T f)^{\circ}$; that is,

$$
\forall \mathfrak{y} \in T Y \forall y \in Y \quad b(\mathfrak{y}, y)=\bigvee_{\substack{\mathfrak{x} \in T f^{-1}(y) \\ x \in f^{-1}(y)}} a(\mathfrak{x}, \mathfrak{y})
$$

Finally, combining the previous results, we have that:

THEOREM 2.4. Let $\mathbf{V}$ be a Heyting algebra and $T:$ Set $\rightarrow$ Set preserve BCsquares. For a class $\&$ of morphisms in $\operatorname{Alg}(T, e ; \mathbf{V}), \mathcal{E} \cap \operatorname{Alg}(T ; \mathbf{V})$ is a class of effective descent morphisms in $\mathrm{Alg}(\mathrm{T} ; \mathbf{V})$ provided that

(1) each $f$ in $\& \cap \operatorname{Alg}(T ; \mathbf{V})$ is a regular epimorphism in $\operatorname{Alg}(T, e ; \mathbf{V})$; 
(2) $\mathcal{E}$ is stable under pullbacks, and

(3) $\operatorname{Alg}(\mathrm{T} ; \mathbf{V})$ is closed under $\mathbb{E}$-images in $\operatorname{Alg}(T, e ; \mathbf{V})$.

\section{3. *-Quotient and Effective Descent Morphisms in $A \lg (\mathrm{T} ; \mathrm{V})$}

Following [13], we call a morphism $f:(X, a) \rightarrow(Y, b)$ in $\operatorname{Alg}(T, e ; \mathbf{V})$ a *-quotient morphism if it satisfies the following condition:

$$
\begin{aligned}
& \forall \mathfrak{Y} \in T^{2} Y \forall \mathfrak{y} \in T Y \forall y \in Y \\
& T b(\mathfrak{Y}, \mathfrak{y}) \otimes b(\mathfrak{y}, y)=\bigvee_{\substack{\mathfrak{X} \in T^{2} f^{-1}(\mathfrak{y}) \\
\mathfrak{x} \in f^{-1}(\mathfrak{y}) \\
\mathfrak{x} \in f^{-1}(\mathfrak{y})}} T a(\mathfrak{X}, \mathfrak{x}) \otimes a(\mathfrak{x}, x) .
\end{aligned}
$$

PROPOSITION 3.1. If $f$ is $a *^{*}$-quotient morphism in $\operatorname{Alg}(\mathrm{T} ; \mathbf{V})$, then it is a regular epimorphism in $\operatorname{Alg}(T, e ; \mathbf{V})$.

Proof. We only have to prove that $b \leq f \cdot a \cdot(T f)^{\circ}$.

Let $\mathfrak{y} \in T Y$ and $y \in Y$. We first remark that, since $T$ commutes with the involution, $T\left(b \cdot e_{Y}\right)=T b \cdot T e_{Y}$. Hence, there exists $I \rightarrow T b\left(T e_{Y}(\mathfrak{y}), \mathfrak{y}\right)$. We thus have

$$
\begin{aligned}
b(\mathfrak{y}, y) & \leq T b\left(T e_{Y}(\mathfrak{y}), \mathfrak{y}\right) \otimes b(\mathfrak{y}, y) \\
& =\bigvee_{\substack{x: T^{2} f(\mathfrak{x})=T e_{Y}(\mathfrak{y}) \\
\mathfrak{x} \in T f^{-1}(\mathfrak{y}) \\
x \in f^{-1}(\mathfrak{y})}} \operatorname{Ta}(\mathfrak{X}, \mathfrak{x}) \otimes a(\mathfrak{x}, x) \quad(f \text { is *-quotient }) \\
& \leq \bigvee_{\substack{\mathfrak{x}: T^{2} f(\mathfrak{x})=T e_{Y}(\mathfrak{h}) \\
x \in f^{-1}(y)}} a\left(m_{X}(\mathfrak{X}), x\right) \quad(a \text { is transitive }) \\
& \leq \bigvee_{\substack{\mathfrak{x} \in T f^{-1}(\mathfrak{y}) \\
x \in f^{-1}(\mathfrak{y})}} a(\mathfrak{x}, x),
\end{aligned}
$$

since, for $\mathfrak{X} \in T^{2} X$ such that $T^{2} f(\mathfrak{X})=T e_{Y}(\mathfrak{y}), T f\left(m_{X}(\mathfrak{X})\right)=m_{Y}\left(T^{2} f(\mathfrak{X})\right)=$ $m_{Y}\left(T e_{Y}(\mathfrak{y})\right)=\mathfrak{y}$.

PROPOSITION 3.2. $\quad \operatorname{Alg}(\mathrm{T} ; \mathbf{V})$ is closed under (*-quotient)-images in $\operatorname{Alg}(T, e ; \mathbf{V})$.

Proof. Let $f:(X, a) \rightarrow(Y, b)$ be a *-quotient morphism in $\operatorname{Alg}(T, e ; \mathbf{V})$ with $(X, a) \in \operatorname{Alg}(\mathrm{T} ; \mathbf{V})$. We want to check that $(Y, b)$ is a transitive lax algebra. For each $\mathfrak{Y} \in T^{2} Y, \mathfrak{y} y \in T Y$ and $y \in Y$,

$$
T b(\mathfrak{Y}, \mathfrak{y}) \otimes b(\mathfrak{y}, y)=\bigvee_{\substack{\mathfrak{x} \in T^{2} f^{-1}(\mathfrak{y}) \\ \mathfrak{x} \in T f^{-1}(\mathfrak{y}) \\ \mathfrak{y} \in f^{-1}(\mathfrak{y})}} \operatorname{Ta}(\mathfrak{X}, \mathfrak{x}) \otimes a(\mathfrak{x}, x)
$$




$$
\begin{aligned}
& \leq \bigvee_{\substack{\mathfrak{X} \in T^{2} f^{-1}(\mathfrak{y}) \\
x \in f^{-1}(\mathfrak{y})}} a\left(m_{X}(\mathfrak{X}), x\right) \\
& \leq b\left(m_{Y}(\mathfrak{Y}), y\right) .
\end{aligned}
$$

THEOREM 3.3. A morphism in $\mathrm{Alg}(\mathrm{T} ; \mathbf{V})$ is an effective descent morphism provided that it is a pullback-stable *-quotient morphism in $\operatorname{Alg}(T, e ; \mathbf{V})$.

Proof. It follows from Theorem 2.4 and Propositions 3.1 and 3.2.

\section{Open and Proper Surjections Are Effective Descent Morphisms}

We define open and proper morphisms in the context of lax algebras using as role models open and proper continuous maps in Top. For that, we recall that, denoting by $U$ the ultrafilter functor, a continuous map $f: X \rightarrow Y$ is proper if and only if, for each ultrafilter $\mathfrak{x}$ on $X$ and each $y \in Y$ with $U f(\mathfrak{x}) \rightarrow y$, there exists $x \in X$ such that $f(x)=y$ and $\mathfrak{x} \rightarrow x$; dually, $f$ is open if and only if, for each $x \in X$ and each $\mathfrak{y}$ on $Y$ such that $\mathfrak{y} \rightarrow f(x)$, there exists an ultrafilter $\mathfrak{x}$ on $X$ such that $U f(\mathfrak{x})=\mathfrak{y}$ and $\mathfrak{x} \rightarrow x$ ([1,7], see also [2]).
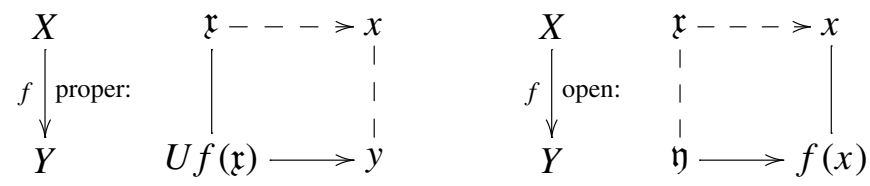

The characterizations above lead naturally to

DEFINITION 4.1. A lax homomorphism $f:(X, a) \rightarrow(Y, b)$ is called proper (open) if the diagram

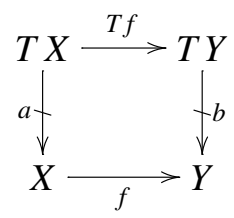

commutes (is a BC-square).

Explicitly, a morphism $f:(X, a) \rightarrow(Y, b)$ is proper if

$$
\forall \mathfrak{x} \in T X \forall y \in Y \quad b(T f(\mathfrak{x}), y)=\bigvee_{x \in f^{-1}(y)} a(\mathfrak{x}, x) ;
$$

and open if

$$
\forall \mathfrak{y} \in T Y \forall x \in X \quad b(\mathfrak{y}, f(x))=\bigvee_{\mathfrak{x} \in T f^{-1}(\mathfrak{y})} a(\mathfrak{x}, x) .
$$


From both equations above we obtain the equality

$$
\forall \mathfrak{y} \in T Y \forall y \in Y \quad b(\mathfrak{y}, y)=\bigvee_{\substack{\mathfrak{x} \in T f^{-1}(\mathfrak{y}) \\ x \in f^{-1}(y)}} a(\mathfrak{x}, x)
$$

in case the map $f: X \rightarrow Y$ is surjective; hence open and proper surjections (i.e. surjective as maps) are regular epimorphisms in $\operatorname{Alg}(T, e ; \mathbf{V})$.

PROPOSITION 4.2. Let $\mathbf{V}$ be a Heyting algebra. Then:

(1) Proper morphisms are pullback-stable in $\operatorname{Alg}(T, e ; \mathbf{V})$.

(2) If $T:$ Set $\rightarrow$ Set preserves BC-squares, then open morphisms are pullbackstable in $\operatorname{Alg}(T, e ; \mathbf{V})$.

Proof. Let

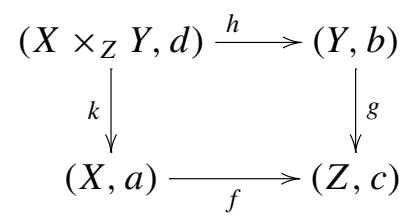

be a pullback in $\operatorname{Alg}(T, e ; \mathbf{V})$. We first recall that, as observed in [5], the pullback structure $d$ is obtained as the meet of $a$ and $b$; that is, for each $\mathfrak{w} \in T\left(X \times{ }_{Z} Y\right)$ and $(x, y) \in X \times{ }_{Z} Y$,

$$
d(\mathfrak{w},(x, y))=a(T k(\mathfrak{w}), x) \wedge b(T h(\mathfrak{w}), y) .
$$

Assume first that $f$ is proper and let $y \in Y$ and $\mathfrak{w} \in T\left(X \times_{Z} Y\right)$. Then

$$
\begin{aligned}
b(T h(\mathfrak{w}), y) \leq & c(T(g \cdot h)(\mathfrak{w}), g(y)) \\
= & c(T f(T k(\mathfrak{w})), g(y)) \\
= & \bigvee_{x: f(x)=g(y)} a(T k(\mathfrak{w}), x) \quad(f \text { is proper })
\end{aligned}
$$

and therefore

$$
\begin{aligned}
& \bigvee_{x: f(x)=g(y)} d(\mathfrak{w},(x, y)) \\
& =\bigvee_{x: f(x)=g(y)} a(T k(\mathfrak{w}), x) \wedge b(T h(\mathfrak{w}), y) \\
& =\left(\bigvee_{x: f(x)=g(y)} a(T k(\mathfrak{w}), x)\right) \wedge b(T h(\mathfrak{w}), y) \quad(\mathbf{V} \text { is l.c.c. }) \\
& =b(T h(\mathfrak{w}), y) .
\end{aligned}
$$

If $f$ is open, we obtain analogously, for any $(x, y) \in X \times_{Z} Y$ and $\mathfrak{y} \in T Y$,

$$
b(\mathfrak{y}, y) \leq \bigvee_{\mathfrak{x}: T f(\mathfrak{x})=T g(\mathfrak{y})} a(\mathfrak{x}, x)
$$


and

$$
\begin{aligned}
& \bigvee_{\mathfrak{w}: T h(\mathfrak{w})=\mathfrak{y}} d(\mathfrak{w},(x, y)) \\
& =\bigvee_{\mathfrak{w}: T h(\mathfrak{w})=\mathfrak{y}} a(T k(\mathfrak{w}), x) \wedge b(\mathfrak{y}, y) \\
& =\left(\bigvee_{\mathfrak{w}: T h(\mathfrak{w})=\mathfrak{y}} a(T k(\mathfrak{w}), x)\right) \wedge b(\mathfrak{y}, y) \quad(\mathbf{V} \text { is l.c.c. }) \\
& =\left(\bigvee_{\mathfrak{x}: T f(\mathfrak{x})=T g(\mathfrak{y})} a(\mathfrak{x}, x)\right) \wedge b(\mathfrak{y}, y) \quad(T \text { preserves BC-squares }) \\
& =b(\mathfrak{y}, y)
\end{aligned}
$$

LEMMA 4.3. Assume that $T(f \cdot r)=T f \cdot \operatorname{Tr}$ for any map $f$. Then with $f$ : $(X, a) \rightarrow(Y, b)$ proper (open) also $T f:(T X, T a) \rightarrow(T Y, T b)$ is proper (open). Proof. It is straightforward.

Recall that, since $T$ preserves the involution, $T$ preserves composition with maps on the right. Hence the hypothesis of the lemma above implies that $T$ is functorial regarding composition with maps (on the left and on the right), as well as regarding composition with map transposes. In particular, $T$ preserves BC-squares.

PROPOSITION 4.4. If $T(f \cdot r)=T f \cdot \operatorname{Tr}$ for any map $f$, then proper and open surjections are *-quotient morphisms.

Proof. It follows from the lemma above and the fact that the tensor product commutes with coproducts in $\mathbf{V}$.

Combining our results we obtain

THEOREM 4.5. Let $\mathbf{V}$ be a complete Heyting algebra, equipped with a tensor product so that $\mathbf{V}$ becomes a symmetric-monoidal closed category. Let $\mathrm{T}$ be a monad on Set extended to Mat $(\mathbf{V})$ such that

$$
T\left(r^{\circ}\right)=(T r)^{\circ} \quad \text { and } \quad T(f \cdot r)=T f \cdot T r
$$

for every $r$ and every map $f$. Then open and proper surjections are effective descent morphisms in $\mathrm{Alg}(\mathrm{T} ; \mathbf{V})$.

As corollaries of this result we obtain that, in Top:

- open surjections are effective descent morphisms, result showed by Sobral (and published in [14]), that can also be deduced from Moerdijk's axioms [11];

- proper surjections are effective descent morphisms, proved by Moerdijk in [12]. 


\section{Effective Descent Morphisms in V-Categories}

In case the monad is the identity monad, reflexive and transitive lax algebras are $\mathbf{V}$-enriched categories as described by Lawvere in [10], while the category of reflexive lax algebras is the category of reflexive $\mathbf{V}$-graphs. The identity functor trivially preserves BC-squares, so that in order to apply the results of Section 3 we only need to assume that $\mathbf{V}$ is a Heyting algebra. The aim of this section is to prove further properties of effective descent morphisms in the category of $\mathbf{V}$ categories. As a final product we obtain that, when the tensor product and the categorical product coincide, the effective descent $\mathbf{V}$-functors are exactly the $*$-quotient morphisms.

LEMMA 5.1. Let $f:(X, a) \rightarrow(Y, b)$ be a $\mathbf{V}$-functor such that its change of base functor $f^{*}$ reflects isomorphisms. Then $f$ is a regular epimorphism in the category of reflexive $\mathbf{V}$-graphs.

Proof. Let $f:(X, a) \rightarrow(Y, b)$ be a $\mathbf{V}$-functor such that $f^{*}$ reflects isomorphisms. Let $y_{0}, y_{1} \in Y$ be given. Then

$$
\alpha:=\bigvee_{x_{i} \in f^{-1}\left(y_{i}\right)} a\left(x_{1}, x_{0}\right) \leq b\left(y_{1}, y_{0}\right)=: \beta
$$

We define ( $\mathbf{V}$-categorical) structures $b_{\alpha}$ and $b_{\beta}$ on $2=\{0,1\}$ as follows:

$$
\begin{aligned}
& b_{\alpha}(1,0)=\alpha, \quad b_{\beta}(1,0)=\beta, \\
& b_{\alpha}(1,1)=b_{\beta}(1,1)=I=b_{\alpha}(0,0)=b_{\beta}(0,0),
\end{aligned}
$$

and we form the pullbacks of $g:\left(2, b_{\alpha}\right) \rightarrow(Y, b)$ and $g:\left(2, b_{\beta}\right) \rightarrow(Y, b)$, with $g(i)=y_{i}(i=0,1)$. The image under $f^{*}$ of the identity map $\left(2, b_{\alpha}\right) \rightarrow\left(2, b_{\beta}\right)$ is an isomorphism, and the conclusion follows.

PROPOSITION 5.2. Every effective descent morphism in $\mathrm{Alg}(\mathrm{Id} ; \mathbf{V})$ is a *-quotient morphism.

Proof. Let $f:(X, a) \rightarrow(Y, b)$ be an effective descent morphism in $\mathrm{Alg}(\mathrm{Id} ; \mathbf{V})$. From the lemma above we know that $f$ is a regular epimorphism and hence an effective descent morphism in $\operatorname{Alg}(\operatorname{Id}, \mathrm{id} ; \mathbf{V})$. We want to show that $f$ is a *-quotient morphism, that is,

$$
\forall y_{0}, y_{1}, y_{2} \in Y \quad b\left(y_{2}, y_{1}\right) \otimes b\left(y_{1}, y_{0}\right)=\bigvee_{\substack{x_{i} \in f^{-1}\left(y_{i}\right) \\ i=0,1,2}} a\left(x_{2}, x_{1}\right) \otimes a\left(x_{1}, x_{0}\right) .
$$

Assume that there exist three elements $y_{0}, y_{1}, y_{2} \in Y$ such that

$$
\alpha:=\bigvee_{x_{i} \in f^{-1}\left(y_{i}\right)} a\left(x_{2}, x_{1}\right) \otimes a\left(x_{1}, x_{0}\right)<b\left(y_{2}, y_{1}\right) \otimes b\left(y_{1}, y_{0}\right) \leq b\left(y_{2}, y_{0}\right) .
$$

Define on $B=\{0,1,2\}$ the following reflexive and non-transitive structure $b_{0}$ : 
- $b_{0}(2,1)=b\left(y_{2}, y_{1}\right), b_{0}(1,0)=b\left(y_{1}, y_{0}\right), b_{0}(2,0)=\alpha$,

- $b_{0}(2,2)=b_{0}(1,1)=b_{0}(0,0)=I$, and

- $b_{0}(i, j):=0$ elsewhere,

so that the map $g:\left(B, b_{0}\right) \rightarrow(Y, b)$, assigning $y_{j}$ to each $j$, is a lax homomorphism. Consider the following pullback diagram in $\mathrm{Alg}(\mathrm{Id}$, id; $\mathbf{V})$

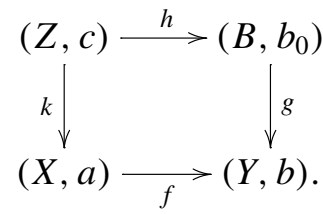

By Theorem 2.1, $(Z, c)$ is not transitive. Hence there exist elements $z_{0}, z_{1}, z_{2}$ of $Z$ such that

$$
c\left(z_{2}, z_{1}\right) \otimes c\left(z_{1}, z_{0}\right) \not \leq c\left(z_{2}, z_{0}\right),
$$

which is only possible if $h\left(z_{i}\right)=i(i=0,1,2)$. For $x_{i}:=k\left(z_{i}\right)$, we have:

- $c\left(z_{2}, z_{0}\right)=a\left(x_{2}, x_{0}\right) \wedge \alpha$, and

- $c\left(z_{2}, z_{1}\right) \otimes c\left(z_{1}, z_{0}\right) \leq a\left(x_{2}, x_{1}\right) \otimes a\left(x_{1}, x_{0}\right)$,

which implies that

$$
a\left(x_{2}, x_{1}\right) \otimes a\left(x_{1}, x_{0}\right) \not \leq a\left(x_{2}, x_{0}\right) \wedge \alpha .
$$

From the inequality $a\left(x_{2}, x_{1}\right) \otimes a\left(x_{1}, x_{0}\right) \leq a\left(x_{2}, x_{0}\right)$, we conclude then that $a\left(x_{2}, x_{1}\right) \otimes a\left(x_{1}, x_{0}\right) \not \leq \alpha$, which contradicts the definition of $\alpha$.

Combining this lemma with Theorem 3.3, we have, for a $\mathbf{V}$-functor $f:(X, a) \rightarrow$ $(Y, b)$ :

$$
\begin{gathered}
f \text { pullback-stable } * \text {-quotient morphism in } \mathrm{Alg}(\mathrm{Id}, \mathrm{id} ; \mathbf{V}) \\
\Downarrow \\
f \text { effective descent morphism in } \mathrm{Alg}(\mathrm{Id} ; \mathbf{V}) \\
\Downarrow \\
f^{*} \text {-quotient morphism }
\end{gathered}
$$

In order to get an equivalence between these three conditions we analyse two situations where *-quotient morphisms are pullback-stable in the category of reflexive $\mathbf{V}$-graphs. The first one is the case $\otimes=\wedge$, that we investigate below. The second case is the subject of the next section.

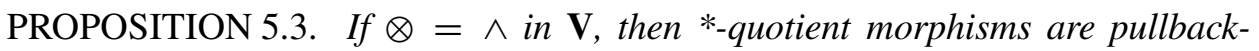
stable in the category of reflexive $\mathbf{V}$-graphs. 
Proof. Let

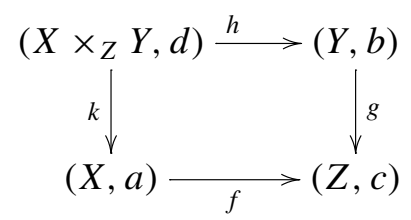

be a pullback in $\operatorname{Alg}(\mathrm{Id}, \mathrm{id} ; \mathbf{V})$ with $f$ a $*$-quotient morphism. For $y_{0}, y_{1}, y_{2} \in Y$, we have

$$
\begin{aligned}
b\left(y_{2}, y_{1}\right) \wedge b\left(y_{1}, y_{0}\right) & \leq c\left(g\left(y_{2}\right), g\left(y_{1}\right)\right) \wedge c\left(g\left(y_{1}\right), g\left(y_{0}\right)\right) \\
& =\bigvee_{x_{i}: f\left(x_{i}\right)=g\left(y_{i}\right)} a\left(x_{2}, x_{1}\right) \wedge a\left(x_{1}, x_{0}\right)
\end{aligned}
$$

and therefore

$$
\begin{aligned}
& b\left(y_{2}, y_{1}\right) \wedge b\left(y_{1}, y_{0}\right) \\
& =\left(\bigvee_{x_{i}: f\left(x_{i}\right)=g\left(y_{i}\right)} a\left(x_{2}, x_{1}\right) \wedge a\left(x_{1}, x_{0}\right)\right) \wedge\left(b\left(y_{2}, y_{1}\right) \wedge b\left(y_{1}, y_{0}\right)\right) \\
& =\bigvee_{x_{i}: f\left(x_{i}\right)=g\left(y_{i}\right)} a\left(x_{2}, x_{1}\right) \wedge b\left(y_{2}, y_{1}\right) \wedge a\left(x_{1}, x_{0}\right) \wedge b\left(y_{1}, y_{0}\right) \\
& =\bigvee_{\left(x_{i}, y_{i}\right) \in h^{-1}\left(y_{i}\right)} d\left(\left(x_{2}, y_{2}\right),\left(x_{1}, y_{1}\right)\right) \wedge d\left(\left(x_{1}, y_{1}\right),\left(x_{0}, y_{0}\right)\right) .
\end{aligned}
$$

Hence we finally obtain:

THEOREM 5.4. If $\mathbf{V}$ is a complete Heyting algebra and $\otimes=\wedge$, then the effective descent morphisms in the category of $\mathbf{V}$-categories are the $*$-quotient morphisms.

\section{Effective Descent Morphisms of (Pre)metric Spaces}

In this section we apply our results to the case $\mathbf{V}=\overline{\mathbb{R}}_{+}$, the half real line $[0,+\infty]$ ordered by $\geq$, with tensor product + . Here the category of $\mathbf{V}$-categories is the category PMet of premetric spaces and non-expansive maps (see [10]).

In this situation we can prove the pullback-stability of *-quotient morphisms in $\operatorname{Alg}(\mathrm{Id}, \mathrm{id} ; \mathbf{V})$, obtaining then:

THEOREM 6.1. A morphism $f:(X, a) \rightarrow(Y, b)$ in PMet is of effective descent if and only if

$$
\forall y_{0}, y_{1}, y_{2} \in Y \quad b\left(y_{2}, y_{1}\right)+b\left(y_{1}, y_{0}\right)=\inf _{x_{i} \in f^{-1}\left(y_{i}\right)} a\left(x_{2}, x_{1}\right)+a\left(x_{1}, x_{0}\right) .(*)
$$


To show pullback-stability of *-quotient morphisms we use the following

LEMMA 6.2. Let $f:(X, a) \rightarrow(Y, b)$ be a non-expansive map in $\mathrm{Alg}\left(\mathrm{Id}, \mathrm{id} ; \overline{\mathbb{R}}_{+}\right)$. For all $y_{0}, y_{1}, y_{2} \in Y$ with $b\left(y_{2}, y_{1}\right) \neq \infty \neq b\left(y_{1}, y_{0}\right)$, the following conditions are equivalent:

(1) $b\left(y_{2}, y_{1}\right)+b\left(y_{1}, y_{0}\right)=\inf _{x_{i} \in f^{-1}\left(y_{i}\right)} a\left(x_{2}, x_{1}\right)+a\left(x_{1}, x_{0}\right)$.

(2) $\forall \varepsilon>0 \forall i=0,1,2 \exists x_{i} \in f^{-1}\left(y_{i}\right):\left\{\begin{array}{l}a\left(x_{2}, x_{1}\right) \leq b\left(y_{2}, y_{1}\right)+\varepsilon \text { and } \\ a\left(x_{1}, x_{0}\right) \leq b\left(y_{1}, y_{0}\right)+\varepsilon \text {. }\end{array}\right.$

Proof. Obviously, condition (2) implies (1). Assume now that (1) holds and let $\varepsilon>0$. Hence there exist $x_{i} \in f^{-1}\left(y_{i}\right)(i=0,1,2)$ such that

$$
b\left(y_{2}, y_{1}\right)+b\left(y_{1}, y_{0}\right)+\varepsilon \geq a\left(x_{2}, x_{1}\right)+a\left(x_{1}, x_{0}\right) .
$$

Therefore,

$$
\begin{aligned}
& a\left(x_{2}, x_{1}\right) \leq b\left(y_{2}, y_{1}\right)+\left(b\left(y_{1}, y_{0}\right)-a\left(x_{1}, x_{0}\right)\right)+\varepsilon \leq b\left(y_{2}, y_{1}\right)+\varepsilon, \quad \text { and } \\
& a\left(x_{1}, x_{0}\right) \leq b\left(y_{1}, y_{0}\right)+\left(b\left(y_{2}, y_{1}\right)-a\left(x_{2}, x_{1}\right)\right)+\varepsilon \leq b\left(y_{1}, y_{0}\right)+\varepsilon .
\end{aligned}
$$

PROPOSITION 6.3. The class of *-quotient morphisms in $\mathrm{Alg}\left(\mathrm{Id}, \mathrm{id} ; \overline{\mathbb{R}}_{+}\right)$is stable under pullbacks.

Proof. Let

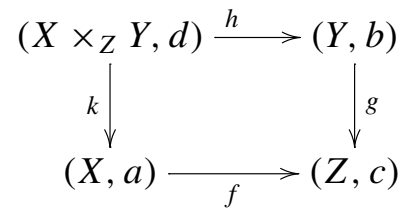

be a pullback in $\operatorname{Alg}\left(\mathrm{Id}, \mathrm{id} ; \overline{\mathbb{R}}_{+}\right)$where $f$ is a $*$-quotient morphism and let $y_{0}, y_{1}, y_{2} \in Y$. The equality of condition $(*)$ is obviously satisfied if one of the distances $b\left(y_{2}, y_{1}\right)$ and $b\left(y_{1}, y_{0}\right)$ is infinite: Hence we may assume that $b\left(y_{2}, y_{1}\right) \neq$ $\infty \neq b\left(y_{1}, y_{0}\right)$. Let $\varepsilon>0$. Since $f$ is a $*$-quotient morphism, by the lemma above there exist $x_{i} \in f^{-1}\left(y_{i}\right)(i=0,1,2)$ such that

$$
\begin{aligned}
& a\left(x_{2}, x_{1}\right) \leq c\left(g\left(y_{2}\right), g\left(y_{1}\right)\right)+\varepsilon \leq b\left(y_{2}, y_{1}\right)+\varepsilon, \quad \text { and } \\
& a\left(x_{1}, x_{0}\right) \leq c\left(g\left(y_{1}\right), g\left(y_{0}\right)\right)+\varepsilon \leq b\left(y_{1}, y_{0}\right)+\varepsilon .
\end{aligned}
$$

This implies

$$
\begin{aligned}
& d\left(\left(x_{2}, y_{2}\right),\left(x_{1}, y_{1}\right)\right)=\max \left\{a\left(x_{2}, x_{1}\right), b\left(y_{2}, y_{1}\right)\right\} \leq b\left(y_{2}, y_{1}\right)+\varepsilon, \quad \text { and } \\
& d\left(\left(x_{1}, y_{1}\right),\left(x_{0}, y_{0}\right)\right)=\max \left\{a\left(x_{1}, x_{0}\right), b\left(y_{1}, y_{0}\right)\right\} \leq b\left(y_{1}, y_{0}\right)+\varepsilon .
\end{aligned}
$$

Finally, the characterization of Theorem $6.1 \mathrm{can}$ be carried out to the category Met of metric spaces (i.e., symmetric and separated premetric spaces with noninfinite distances) and non-expansive maps. For that, we use Theorem 2.1 with $\mathbf{B}=\operatorname{Alg}\left(\mathrm{Id}, \mathrm{id} ; \overline{\mathbb{R}}_{+}\right)$and $\mathbf{A}=$ Met. 
THEOREM 6.4. A morphism $f:(X, a) \rightarrow(Y, b)$ in Met is of effective descent if and only if

$$
\forall y_{0}, y_{1}, y_{2} \in Y \quad b\left(y_{2}, y_{1}\right)+b\left(y_{1}, y_{0}\right)=\inf _{x_{i} \in f^{-1}\left(y_{i}\right)} a\left(x_{2}, x_{1}\right)+a\left(x_{1}, x_{0}\right) .
$$

Finally we point out that effective descent morphisms in Met do not coincide with effective descent morphisms in Top between metric spaces. For instance, the projection $\pi_{1}:(\mathbb{R} \backslash\{0\} \times\{0\}) \cup(\{0\} \times \mathbb{R} \backslash\{0\}) \rightarrow \mathbb{R}$ topologically is not even a biquotient map but it follows easily from our criterion that it is an effective descent morphism in Met.

\section{Acknowledgements}

We thank George Janelidze for valuable discussions on the subject of this paper.

The authors acknowledge partial financial assistance by Centro de Matemática da Universidade de Coimbra/FCT and Unidade de Investigação e Desenvolvimento Matemática e Aplicações da Universidade de Aveiro/FCT.

\section{References}

1. Bourbaki, N.: Topologie Générale, Hermann, Paris, 1961.

2. Clementino, M. M. and Hofmann, D.: Triquotient maps via ultrafilter convergence, Proc. Amer. Math. Soc. 130 (2002), 3423-3431.

3. Clementino, M. M. and Hofmann, D.: Topological features of lax algebras, Appl. Categ. Structures 11 (2003), 267-286.

4. Clementino, M. M. and Hofmann, D.: On extensions of lax monads, Theory Appl. Categories (to appear).

5. Clementino, M. M., Hofmann, D. and Tholen, W.: Exponentiability in categories of lax algebras, Theory Appl. Categories 11 (2003), 337-352.

6. Clementino, M. M. and Tholen, W.: Metric, topology and multicategory - a common approach, J. Pure Appl. Algebra 179 (2003), 13-47.

7. Hájek, O.: Notes on quotient maps, Comment. Math. Univ. Carolinae 7 (1966), 319-323.

8. Janelidze, G. and Sobral, M.: Finite preorders and topological descent I, J. Pure Appl. Algebra 175 (2002), 187-205.

9. Janelidze, G. and Tholen, W.: Facets of descent I, Appl. Categ. Structures 2 (1994), 245-281.

10. Lawvere, F. W.: Metric spaces, generalized logic, and closed categories, Rend. Sem. Mat. Fis. Milano 43 (1973), 135-166.

11. Moerdijk, I.: Descent theory for Toposes, Bull. Soc. Math. Belgique 41 (1989), 373-391.

12. Moerdijk, I.: Descent for proper maps of topological spaces, Manuscript, 1990.

13. Reiterman, J. and Tholen, W.: Effective descent maps of topological spaces, Topology Applications 57 (1994), 53-69.

14. Sobral, M. and Tholen, W.: Effective descent morphisms and effective equivalence relations, in Category Theory 1991, CMS Conference Proceedings 13, Amer. Math. Soc., Providence, RI, 1992, pp. 421-432. 